ANNALES

POLONICI MATHEMATICI

$92.1(2007)$

\title{
Boundary values of functions in Cegrell's class $\mathcal{E}_{\psi}$
}

\author{
by Pham HoAng Hiep (Hanoi)
}

Abstract. We study boundary values of functions in Cegrell's class $\mathcal{E}_{\psi}$.

1. Introduction. Let $\Omega$ be a bounded hyperconvex domain in $\mathbb{C}^{n}$. Denote by $\operatorname{PSH}(\Omega)$ the plurisubharmonic (psh) functions on $\Omega$. The complex Monge-Ampère operator $\left(d d^{c}\right)^{n}$ is well defined over the class of locally bounded psh functions, according to the fundamental work of Bedford and Taylor in [BT1], [BT2]. Cegrell introduced a general class $\mathcal{E}$ of psh functions on which the complex Monge-Ampère operator $\left(d d^{c}\right)^{n}$ can be defined. He obtained many important results of pluripotential theory in the class $\mathcal{E}$, for example, the comparison principle and solvability of the Dirichlet problem (see [Ce1], [Ce2]). Recently, he introduced in [Ce3] a new class $\mathcal{E}_{\psi}$. The main aim of this note is to study boundary values of functions in the class $\mathcal{E}_{\psi}$.

Acknowledgments. This research was done during a visit of the author to Mid Sweden University in Sundsvall, Sweden in 2006. I would like to express my hearty thanks to Professor Urban Cegrell for the kind invitation. I am is also indebted to the referee for his useful comments.

2. Preliminaries. First we recall some elements of pluripotential theory that will be used throughout the paper. All this can be found in [BT2], [Ce1], $[\mathrm{Ce} 2],[\mathrm{Kl}],[\mathrm{Ko}]$.

2.1. The following classes of psh functions were introduced by Cegrell in $[\mathrm{Ce} 1]$ and $[\mathrm{Ce} 2]$ :

$$
\begin{aligned}
& \mathcal{E}_{0}=\mathcal{E}_{0}(\Omega)=\left\{\varphi \in \operatorname{PSH}^{-}(\Omega) \cap L^{\infty}(\Omega): \lim _{z \rightarrow \partial \Omega} \varphi(z)=0, \int_{\Omega}\left(d d^{c} \varphi\right)^{n}<\infty\right\}, \\
& \mathcal{E}_{p}=\mathcal{E}_{p}(\Omega)=\left\{\varphi \in \operatorname{PSH}(\Omega): \exists \mathcal{E}_{0}(\Omega) \ni \varphi_{j} \searrow \varphi, \sup _{j \geq 1} \int_{\Omega}\left(-\varphi_{j}\right)^{p}\left(d d^{c} \varphi_{j}\right)^{n}<\infty\right\},
\end{aligned}
$$

2000 Mathematics Subject Classification: Primary 32W20; Secondary 32 U15.

Key words and phrases: complex Monge-Ampère operator, plurisubharmonic function.

This work was supported by the National Research Program for Natural Sciences, Vietnam. 


$$
\begin{gathered}
\mathcal{F}=\mathcal{F}(\Omega)=\left\{\varphi \in \operatorname{PSH}^{-}(\Omega): \exists \mathcal{E}_{0}(\Omega) \ni \varphi_{j} \searrow \varphi, \sup _{j \geq 1} \int_{\Omega}\left(d d^{c} \varphi_{j}\right)^{n}<\infty\right\}, \\
\mathcal{E}=\mathcal{E}(\Omega)=\left\{\varphi \in \operatorname{PSH}^{-}(\Omega): \exists \varphi_{K} \in \mathcal{F}(\Omega)\right. \text { such that } \\
\left.\varphi_{K}=\varphi \text { on } K, \forall K \subset \subset \Omega\right\} .
\end{gathered}
$$

2.2. For each $\psi \in \mathrm{PSH}^{-}(\Omega), \psi \neq \equiv 0$, Cegrell [Ce3] introduced a new class of psh functions

$$
\begin{aligned}
\mathcal{E}_{\psi}=\mathcal{E}_{\psi}(\Omega)=\left\{\varphi \in \operatorname{PSH}^{-}(\Omega): \exists \mathcal{E}_{0}(\Omega) \ni\right. & \varphi_{j} \searrow \varphi \\
& \left.\sup _{j \geq 1} \int_{\Omega}-\psi\left(d d^{c} \varphi_{j}\right)^{n}<\infty\right\} .
\end{aligned}
$$

By Proposition 3.1 in [Ce3] we have $\mathcal{E}_{\psi} \subset \mathcal{E}$. It is known that if $v \leq u$ and $v \in \mathcal{E}_{\psi}$ then $u \in \mathcal{E}_{\psi}$. By Theorem 5.5 in [Ce2] we have $u+v \in \mathcal{E}_{\psi}$ for all $u, v \in \mathcal{E}_{\psi}$.

2.3. Let $a \in \Omega$. According to Klimek (see $[\mathrm{Kl}]$ ), the pluricomplex Green function with poles at $a$ is defined by

$$
g_{\Omega, a}=g_{a}(z)=\sup \left\{u \in \operatorname{PSH}^{-}(\Omega), u(z)-\log |z-a| \leq O(1) \text { as } z \rightarrow a\right\} .
$$

Demailly [De] proved that $\left(d d^{c} \max \left(g_{a},-\varepsilon\right)\right)^{n}$ is weak*-convergent to a measure $\mu_{\Omega, a}$ supported on $\partial \Omega$ as $\varepsilon \rightarrow 0$. He discovered the following interesting formula:

$$
u(a)=\frac{1}{(2 \pi)^{n}} \int_{\partial \Omega} u d \mu_{\Omega, a}+\frac{1}{(2 \pi)^{n}} \int_{\Omega} g_{a} d d^{c} u \wedge\left(d d^{c} g_{a}\right)^{n-1}
$$

for all $u \in \operatorname{PSH}(\Omega) \cap C(\bar{\Omega})$.

2.4. Let $u \in \operatorname{PSH}^{-}(\Omega)$. We set $u^{*}(\xi)=\limsup _{z \rightarrow \xi} u(z)$ for all $\xi \in \bar{\Omega}$. By the comparison principle for the classes $\mathcal{F} \cup \mathcal{E}_{p}$ we obtain $\left.u^{*}\right|_{\partial \Omega} \equiv 0$ for all $u \in \mathcal{F} \cup \mathcal{E}_{p}$ (see $\left.[\AA \mathrm{h}],[\mathrm{Ce} 1,2],[\AA \mathrm{CH}],[\mathrm{H} 1,2]\right)$. By Theorem 5.8 in [Ce2] we find a function $u \in \mathcal{F}_{1}$ such that $\liminf _{z \rightarrow \xi} u(z)=-\infty$ for all $\xi \in \partial \Omega$.

Next we introduce a result needed for our paper:

2.5. Proposition. Let $u_{j}, v_{j} \in \mathcal{F}, u \in \mathrm{PSH}^{-}(\Omega)$ be such that $u_{j} \searrow u$, $v_{j} \searrow u$. Then

$$
\lim _{j \rightarrow \infty} \int_{\Omega}-\varphi\left(d d^{c} u_{j}\right)^{n}=\lim _{j \rightarrow \infty} \int_{\Omega}-\varphi\left(d d^{c} v_{j}\right)^{n}
$$

for all $\varphi \in \mathrm{PSH}^{-}(\Omega)$.

Proof. For each $k$ we set $w_{j}=\max \left(u_{k}, v_{j}\right)$. Integration by parts gives

$$
\int_{\Omega}-\varphi\left(d d^{c} w_{j}\right)^{n} \leq \int_{\Omega}-\varphi\left(d d^{c} v_{j}\right)^{n}
$$


for all $j \geq 1$. Moreover since $w_{j} \searrow u_{k} \in \mathcal{F}$ as $j \rightarrow \infty$ we obtain

$$
\int_{\Omega}-\varphi\left(d d^{c} u_{k}\right)^{n} \leq \lim _{j \rightarrow \infty} \int_{\Omega}-\varphi\left(d d^{c} v_{j}\right)^{n} .
$$

Letting $k \rightarrow \infty$ we get

$$
\lim _{j \rightarrow \infty} \int_{\Omega}-\varphi\left(d d^{c} u_{j}\right)^{n} \leq \lim _{j \rightarrow \infty} \int_{\Omega}-\varphi\left(d d^{c} v_{j}\right)^{n} .
$$

3. Boundary values of functions in the class $\mathcal{E}_{\psi}$. The main result of the note is the following

3.1. Theorem. Let $\Omega$ be a bounded hyperconvex domain in $\mathbb{C}^{n}(n \geq 2)$ and $u \in \mathcal{E}_{\psi}(\Omega)$ for some $\psi \in \operatorname{PSH}^{-}(\Omega), \psi \neq \equiv$. Then $\int_{\partial \Omega} u^{*} d \mu_{\Omega, a}=0$ for all $a \in \Omega$.

Proof. By the definition of the class $\mathcal{E}_{\psi}$ we find $\mathcal{E}_{0} \ni u_{j} \searrow u$ such that

$$
\sup _{j \geq 1} \int_{\Omega}-\psi\left(d d^{c} u_{j}\right)^{n}<\infty
$$

Let $a \in \Omega$. From

$$
\sup _{z \in \Omega} \frac{\left|\max \left(g_{a}(z),-1\right)\right|}{|\psi(z)|}<\infty
$$

and from Proposition 2.5 we get

$$
A=\sup _{j \geq 1} \int_{\Omega}-\max \left(g_{a},-1\right)\left(d d^{c} \max \left(j g_{a}, u\right)\right)^{n}<\infty .
$$

Let $K$ be a compact subset in $\left\{u^{*}<0\right\} \cap \partial \Omega$. We only have to prove that

$$
\int_{K} d \mu_{\Omega, a}=0 .
$$

Let $s>0$ and $U$ be a neighborhood of $K$ such that $\left.u\right|_{U \cap \Omega}<-s$. We have

$$
\begin{aligned}
\int_{\Omega}-\max \left(g_{a},-1\right)\left(d d^{c} \max \left(j g_{a}, u\right)\right)^{n} \\
\quad=\int_{\left\{j g_{a} \leq u\right\}}-\max \left(g_{a},-1\right)\left(d d^{c} \max \left(j g_{a}, u\right)\right)^{n} \\
\geq \int_{\left\{j g_{a} \leq u\right\}}-\max (u / j,-1)\left(d d^{c} \max \left(j g_{a}, u\right)\right)^{n} \\
=j^{n-1} \int_{\Omega}-\max (u,-j)\left(d d^{c} \max \left(g_{a}, u / j\right)\right)^{n}
\end{aligned}
$$




$$
\begin{aligned}
& \geq j^{n-1} \int_{\Omega}-\max (u,-j)\left(d d^{c} \max \left(g_{a}, u / j,-s / j\right)\right)^{n} \\
& \geq j^{n-1} s \int_{U}\left(d d^{c} \max \left(g_{a}, u / j,-s / j\right)\right)^{n}=j^{n-1} s \int_{U}\left(d d^{c} \max \left(g_{a},-s / j\right)\right)^{n}
\end{aligned}
$$

for $j \geq 1$. Therefore

$$
\int_{U}\left(d d^{c} \max \left(g_{a},-s / j\right)\right)^{n} \leq \frac{A}{j^{n-1} s}
$$

for $j \geq 1$. Moreover, since $\left(d d^{c} \max \left(g_{a},-\varepsilon\right)\right)^{n}$ is weak ${ }^{*}$-convergent to a measure $\mu_{\Omega, a}$ on $\mathbb{C}^{n}$ as $\varepsilon \rightarrow 0$ we obtain

$$
\int_{U} d \mu_{\Omega, a} \leq \liminf _{j \rightarrow \infty} \int_{U}\left(d d^{c} \max \left(g_{a},-s / j\right)\right)^{n} \leq \liminf _{j \rightarrow \infty} \frac{A}{j^{n-1} s}=0 .
$$

Hence

$$
\int_{K} d \mu_{\Omega, a}=0 .
$$

3.2. Corollary. Let $\Omega$ be a bounded B-regular domain in $\mathbb{C}^{n}$ and $u \in$ $\mathcal{E}_{\psi}(\Omega)$ for some $\psi \in \mathrm{PSH}^{-}(\Omega), \psi \not \equiv 0$. Then $\left.u^{*}\right|_{\partial \Omega} \equiv 0$.

Proof. We assume that $u^{*}\left(\xi_{0}\right)<0$ for some $\xi_{0} \in \partial \Omega$. Let $r>0$ be such that

$$
u^{*}(\xi)<u^{*}\left(\xi_{0}\right) / 2
$$

for all $\xi \in B\left(\xi_{0}, r\right) \cap \partial \Omega$. By Theorem 3.1 we have

$$
\int_{B\left(\xi_{0}, r\right)} d \mu_{\Omega, a}=0
$$

for all $a \in \Omega$. Let $f \in C(\partial \Omega)$ be such that $0 \leq f \leq 1, f=1$ on $B\left(\xi_{0}, r / 2\right) \cap$ $\partial \Omega$ and $f=0$ on $\partial \Omega \backslash B\left(\xi_{0}, r\right)$. We find a function $h \in \operatorname{PSH}(\Omega) \cap C(\bar{\Omega})$ such that $\left(d d^{c} h\right)^{n}=0$ and $\left.h\right|_{\partial \Omega}=f$. Let $a \in \Omega$ be such that $h(a)>0$. By [De] we have

$$
\begin{aligned}
h(a) & =\frac{1}{(2 \pi)^{n}} \int_{\partial \Omega} f d \mu_{\Omega, a}+\frac{1}{(2 \pi)^{n}} \int_{\Omega} g_{a} d d^{c} h \wedge\left(d d^{c} g_{a}\right)^{n-1} \\
& \leq \frac{1}{(2 \pi)^{n}} \int_{\partial \Omega} f d \mu_{\Omega, a} \leq \frac{1}{(2 \pi)^{n}} \int_{B\left(\xi_{0}, r\right)} d \mu_{\Omega, a} .
\end{aligned}
$$

Hence

$$
\int_{B\left(\xi_{0}, r\right)} d \mu_{\Omega, a} \geq(2 \pi)^{n} h(a)>0
$$

which contradicts $\int_{B\left(\xi_{0}, r\right)} d \mu_{\Omega, a}=0$. 
3.3. Corollary. Let $\Omega=\Omega_{1} \times \Omega_{2}$ where $\Omega_{1} \subset \mathbb{C}^{n_{1}}, \Omega_{2} \subset \mathbb{C}^{n_{2}}$ are bounded $B$-regular domains and $u \in \mathcal{E}_{\psi}(\Omega)$ for some $\psi \in \mathrm{PSH}^{-}(\Omega), \psi \neq \equiv 0$. Then $\left.u^{*}\right|_{\partial \Omega_{1} \times \partial \Omega_{2}} \equiv 0$.

Proof. From $g_{\Omega,\left(a_{1}, a_{2}\right)}=\max \left(g_{\Omega_{1}, a_{1}}, g_{\Omega_{2}, a_{2}}\right)$ and from Theorem 7 in $[\mathrm{B} \nmid 2]$ we get

$$
\mu_{\Omega,\left(a_{1}, a_{2}\right)}=\mu_{\Omega_{1}, a_{1}} \times \mu_{\Omega_{2}, a_{2}}
$$

for all $\left(a_{1}, a_{2}\right) \in \Omega_{1} \times \Omega_{2}$. By this formula and a copy of the proof of Corollary 3.2 we infer that $\left.u^{*}\right|_{\partial \Omega_{1} \times \partial \Omega_{2}} \equiv 0$.

Let $\Omega_{1}, \Omega_{2}$ be bounded hyperconvex domains in $\mathbb{C}$. We construct a function $u \in \mathcal{E}_{\psi}\left(\Omega_{1} \times \Omega_{2}\right)$ for some $\psi \in \operatorname{PSH}^{-}\left(\Omega_{1} \times \Omega_{2}\right), \psi \not \equiv 0$ such that $\left.u^{*}\right|_{\partial \Omega_{1} \times \Omega_{2} \cup \Omega_{1} \times \partial \Omega_{2}}<0$ :

3.4. Proposition. Let $\Omega=\Omega_{1} \times \Omega_{2}$ where $\Omega_{1}, \Omega_{2}$ are bounded hyperconvex domains in $\mathbb{C}$. Then $\max \left(g_{\Omega_{1}, a_{1}},-1\right)+\max \left(g_{\Omega_{2}, a_{2}},-1\right) \in \mathcal{E}_{\psi}(\Omega)$ with $\psi=\max \left(g_{\Omega_{1}, a_{1}}, g_{\Omega_{2}, a_{2}}\right)$ for all $\left(a_{1}, a_{2}\right) \in \Omega_{1} \times \Omega_{2}$.

Proof. By Theorem 5.5 in [Ce2] we only have to prove that $u=$ $\max \left(g_{\Omega_{1}, a_{1}},-1\right) \in \mathcal{E}_{\psi}(\Omega)$. Set

$$
u_{j}=\max \left(g_{\Omega_{1}, a_{1}}, j g_{\Omega_{2}, a_{2}}-1\right) .
$$

Then $\mathcal{E}_{0}(\Omega) \ni u_{j} \searrow u$. By Theorem 7 in [Bł2] we have

$$
\begin{aligned}
\int_{\Omega}-\psi( & \left.d d^{c} u_{j}\right)^{2}=\int_{\Omega}-\psi d d^{c}\left(g_{\Omega_{1}, a_{1}},-1\right) \wedge d d^{c}\left(j g_{\Omega_{2}, a_{2}},-1\right) \\
= & \int_{\left\{g_{\Omega_{1}, a_{1}}=-1\right\} \times\left\{g_{\Omega_{2}, a_{2}}=-1 / j\right\}}-j \psi d d^{c}\left(g_{\Omega_{1}, a_{1}},-1\right) \wedge d d^{c}\left(g_{\Omega_{2}, a_{2}},-1 / j\right) \\
= & \int_{\left\{g_{\Omega_{1}, a_{1}}=-1\right\} \times\left\{g_{\Omega_{2}, a_{2}}=-1 / j\right\}} d d^{c}\left(g_{\Omega_{1}, a_{1}},-1\right) \wedge d d^{c}\left(g_{\Omega_{2}, a_{2}},-1 / j\right) \\
= & \int_{\Omega_{1}} d d^{c}\left(g_{\Omega_{1}, a_{1}},-1\right) \int_{\Omega_{1}} d d^{c}\left(g_{\Omega_{2}, a_{2}},-1 / j\right)=(2 \pi)^{2} .
\end{aligned}
$$

Hence $u \in \mathcal{E}_{\psi}(\Omega)$.

\section{References}

[Åh] P. Åhag, The complex Monge-Ampère operator on bounded hyperconvex domains, Ph.D. thesis, Umeå Univ., 2002.

[ÅCH] P. Åhag, R. Czyż and Pham Hoang Hiep, Concerning the energy class $\mathcal{E}_{p}$ for $0<p<1$, Ann. Polon. Math. 91 (2007), to appear.

[Bł1] Z. Błocki, The complex Monge-Ampère operator in hyperconvex domains, Ann. Scuola Norm. Sup. Pisa 23 (1996), 721-747. 
[Bł2] Z. Błocki, Equilibrium measure of a product subset of $\mathbb{C}^{n}$, Proc. Amer. Math. Soc. 128 (2000), 3595-3599.

[BT1] E. Bedford and B. A. Taylor, The Dirichlet problem for the complex MongeAmpère operator, Invent. Math. 37 (1976), 1-44.

[BT2] - - - A new capacity for plurisubharmonic functions, Acta Math. 149 (1982), $1-40$.

[Ce1] U. Cegrell, Pluricomplex energy, ibid. 180 (1998), 187-217.

[Ce2] - , The general definition of the complex Monge-Ampère operator, Ann. Inst. Fourier (Grenoble) 54 (2004), 159-179.

[Ce3] -, Two examples in pluripotential theory, Dept. of Math., Mid-Sweden Univ., Research Report no. 14 (2000).

[De] J.-P. Demailly, Mesures de Monge-Ampère et mesures pluriharmoniques, Math. Z. 194 (1987), 519-564.

[Kl] M. Klimek, Pluripotential Theory, Oxford Univ. Press, New York, 1991.

[Ko] S. Kołodziej, The range of the complex Monge-Ampère operator, II, Indiana Univ. Math. J. 44 (1995), 765-782.

[H1] Pham Hoang Hiep, A characterization of bounded plurisubharmonic functions, Ann. Polon. Math. 85 (2005), 233-238.

[H2] - The comparison principle and Dirichlet problem in the class $\mathcal{E}_{p}(f), p>0$, ibid. 88 (2006), 247-261.

Department of Mathematics

Hanoi University of Education (Dai Hoc Su Pham HaNoi)

Cau Giay, Hanoi, VietNam

E-mail: phhiep_vn@yahoo.com

Received 27.12.2006

and in final form 15.2.2007 